\title{
The SKA Approach to Sustainable Research
}

\author{
Simon T. Berry
}

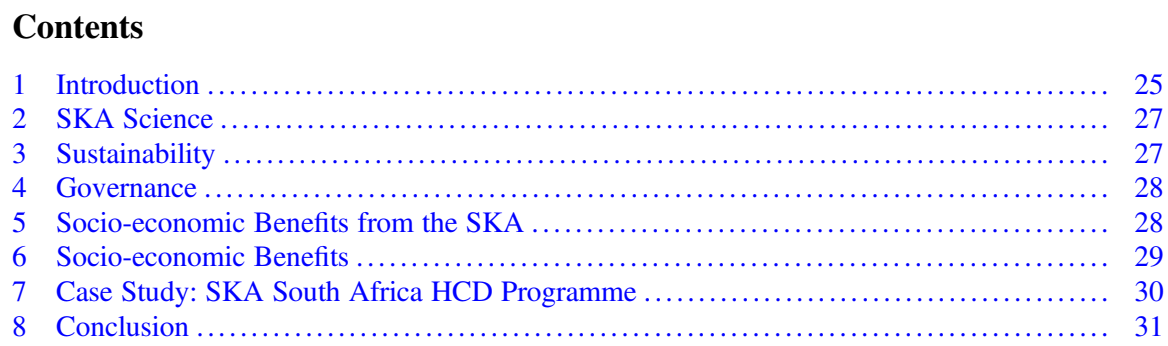

\section{Introduction}

The Square Kilometre Array (SKA) is an ambitious project to build a research infrastructure that will enable breakthrough science and discoveries not otherwise possible with current or planned astronomy facilities. Comprising two radio telescopes it will ultimately be the largest scientific instrument on Earth, both in physical scale and in terms of the volume of data it will generate. Like all infrastructures, there is a requirement for SKA to demonstrate where benefits have been realised from past investment, and the potential for future ongoing benefits from technology and knowledge transfer and innovations. The essay discusses the range of non-science benefits for the funders and for wider society going beyond SKA's core scientific mission and the challenges of thinking about regional impacts when designing a global research infrastructure.

SKA will have a uniquely distributed character: one observatory, operating two telescopes, on three continents for the global scientific community. The SKA's operational phase is expected to last at least 50 years.

\footnotetext{
S. T. Berry $(\bowtie)$

Director of Corporate Strategy, SKA Organisation, Macclesfield, United Kingdom e-mail: S.Berry@ skatelescope.org 
The recently completed SKA Global Headquarters is located at Jodrell Bank near Manchester in the UK, home to the organisation - the future SKA Observatorythat oversees development, construction and operations. The two other SKA sites are radio quiet zones and home to the telescopes themselves: a mid-frequency array in South Africa (SKA-mid), and a low-frequency array in Australia (SKA-low). In order to take advantage of the development of computing and other innovative technologies of relevance for the SKA programme, the construction of the SKA will be phased. Work is currently focused on the first phase named SKA1.

SKA1 will be a transformational scientific facility. It will tackle some of the most fundamental scientific questions of our time, ranging from the birth of the Universe to the origins of life.

To do so, the SKA will collect unprecedented amounts of data, requiring supercomputers amongst the world's fastest to process this data in near real time, before turning these into science products for distribution around the world through a network of SKA Regional Centres located in partner countries. Those data centres will be the final interface with the end users - the scientists-who will turn these science products into information, and finally knowledge. The two SKA telescopes differ in design and are complementary by their very nature. Both are interferometers: arrays of antennas which when linked together act as one enormous telescope, bigger than would ever be possible in a traditional single-dish design.

In South Africa, the Design Baseline for SKA1-mid comprises 197 dishes, 64 of which are already in place and form the MeerKAT precursor telescope, itself a world-class facility, which will be integrated into SKA1-mid. In Western Australia, over 130,000 low-frequency antennas will form SKA1-low, spread across 512 antenna stations.

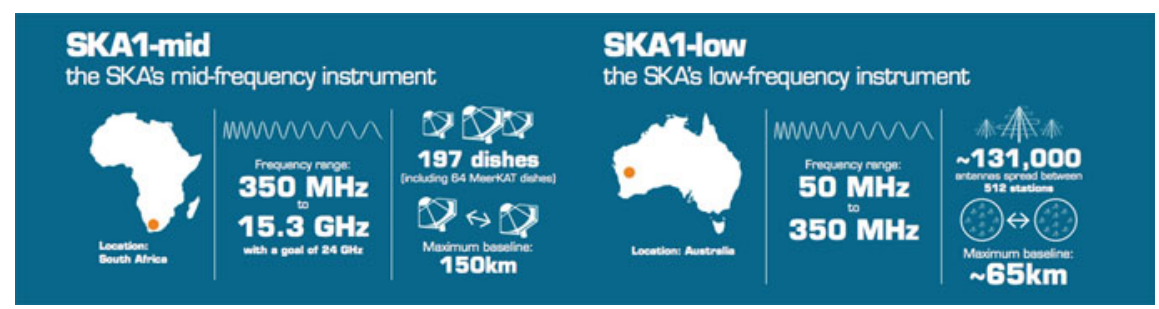

Infographics describing SKA1-mid and SKA1-low telescopes

The telescopes' design is scalable and upgradable, allowing future improvements to maintain their world-leading capabilities, and also to align with available funding. This includes state-of-the art scientific and computing infrastructures, designed to progressively exploit the capabilities of the Observatory as computing technology continuously improves over the coming decades. 


\section{SKA Science}

The SKA's science goals are broad and ambitious, looking back into the history of the Universe as far as the Cosmic Dawn, when the very first stars and galaxies formed, in order to seek answers to some of the biggest remaining questions in astrophysics. Among them: How do galaxies evolve? What is dark energy and what role does it play in the expansion of the Universe? What causes planets to form around stars? Can we find and understand where gravitational waves come from? Is there life out there? Individually, and working together with other next-generation facilities, SKA will deliver a profound impact.

The SKA will devote between 50 and $75 \%$ of its time to Key Science Projectsmajor surveys that require lots of observing time on the telescope, focused on making ground-breaking discoveries. The remainder will be allocated to traditional, smaller-scale studies depending on scientific and technical feasibility. Access to the telescopes will be on merit, but with the majority of time being reserved for scientists from the Observatory's member countries.

As with any major scientific endeavour, the SKA's design has undergone changes during the course of the project to take account of technological evolution and funding constraints. In July 2013, prior to the start of detailed design, the SKA Organisation Board set a cost cap of $€ 650 \mathrm{~m}$ for the construction of SKA1. This has been periodically adjusted for inflation and, based on the most recent adjustment (December 2017), is set at €691 m, representing the 'Deployment Baseline' of the infrastructure. As of June 2019, the cost estimate for SKA1's Design Baseline, which is the agreed design for the first phase of the SKA project described above, is $€ 914 \mathrm{~m}(2017 €)$. The cost estimate is under regular review and will be subject to a full cost audit in conjunction with the Critical Design Review of the overall SKA1 system. Over the coming months, alongside the implementation of the organisation to deliver SKA, discussions will focus on available resources against the project cost to establish a final project scope.

A high-level view of the technical and scientific milestones for SKA Phase 1 is captured in the figure below.

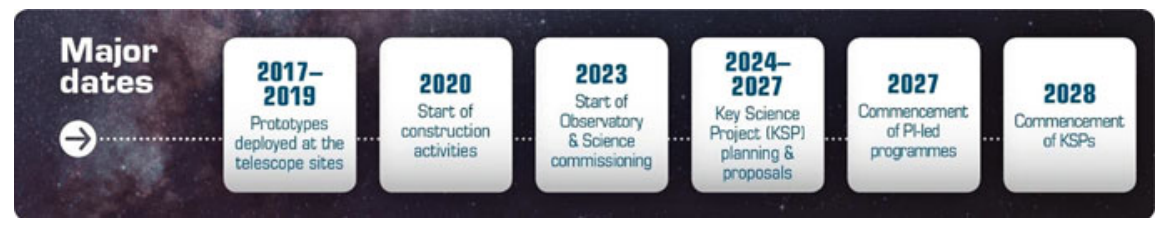

\section{Sustainability}

SKA has worked intensively to align itself with the outputs of the ESFRI working group on RI Sustainability. Sustainability for the SKA means a combination of financial stability, a governance environment that will endure through the project's 
lifetime, and a series of measures that ensures SKA sits sustainably in its host countries and delivers a compelling socio-economic benefits case for its membership.

\section{Governance}

Following a familiar route for European Research Infrastructures, SKA has evolved from an informal collaboration through to the establishment of a (UK) company to manage the design effort preparing for construction. Then, following a decision taken by the SKA member governments in 2015, work began to develop an intergovernmental organisation (the SKA Observatory) to manage the construction and operation of the facility. Enabling a long term, sustainable governance structure has been at the heart of the decision.

The IGO will operate in a similar way to other long-established intergovernmental infrastructure organisations such as CERN, ESA and ESO and is being designed taking into account best practice from all relevant European and global examples. Such organisations embody a commitment by the Members to the long-term financial and operational arrangements that are essential for the secure delivery of large complex research programmes and facilities. It will move from its present status to that of a treaty-level organisation registered with the United Nations in early 2020, and will be governed by a Council appointed by the Members and acting collectively to advance the project in accordance with the principles set out in the Convention that will be its founding document. It will be established and will operate under international law, with the appropriate legal status and privileges in the Member countries.

\section{Socio-economic Benefits from the SKA}

The creation of large scientific facilities, while driven by clear and compelling scientific needs, leads not only to scientific advances but also to a range of non-science benefits for the funders and for wider society. The 2015 report by "Science Business" entitled "Big Science: What's it Worth?" identified a range of benefits arising from investment in "Big Science", from capacitive touch screens to cochlear implants, and also presented a set of case studies looking in more depth at the impact of Big Science projects (including SKA) beyond their brief. A variety of other studies have also addressed the issue of non-science benefits arising from the major investment entailed in such facilities. Like all infrastructures, there is pressure on SKA to demonstrate where benefits have been realised from past investment, and the potential for future ongoing benefits from technology and knowledge transfer and innovations.

From the earliest ideas, and throughout the development of the SKA concept, close attention has been paid to the potential non-science benefits of the Project. The 
overall aim is to ensure that there is a progressive and persistent case for investment that goes beyond the science case, based on innovation and industrial return. The evolving design and construction plans endeavour to facilitate identification, exploitation and propagation of innovation and other non-science benefits. Moreover, SKA has a potentially unique opportunity to establish a structure for identifying and tracking all relevant metrics for impact from outset to conclusion.

The first and most immediate benefits from investment in SKA are guaranteed access to direct contracts and the opportunity to make in-kind contributions, both of which are only open to Members of the SKA Observatory. Additionally, the very fact of working with such an advanced project can lead to companies developing existing and new skills that enable them to be more competitive. Long-established evidence from companies working with other cutting-edge facilities (e.g. CERN*) has shown that responding to the exacting standards demanded has raised their game and qualified them to bid for other high-technology contracts, thus extending their markets.

\section{Socio-economic Benefits}

The demands of the SKA will help drive technology development, and contribute to and be a stimulant for invention and innovation within global industries. In conjunction with market forces, they will lead to benefits to society that will be widely applicable in other established fields and will begin even before the astronomical discoveries that will eventually be made using the telescope. Countries that participate in the SKA could reap considerable socio-economic benefits by participating at the forefront of these developments. Spin-off innovations in areas specific to the SKA's computing activities will, through the industries with which SKA will be working to develop them, benefit other systems that process large volumes of data from geographically dispersed sources. Potential areas where innovations inspired by the SKA's needs could have wider applicability include data management techniques, data mining and analytics, imaging algorithms, remote visualisation and pattern matching (all of which will have impact in areas such as medicine, transport and security).

The significant contribution that the SKA will make to new developments across a range of technologies will also benefit the global knowledge-based economy beyond scientific research and academia. For the first time, the developing world is an active and integral contributor to fundamental research on an unprecedented scale. Emerging and developing countries are already active in the production and exchange of knowledge, stimulating their participation in the global knowledgebased economy. In addition, the existence of the facility will create demand leading to employment opportunities such as visitors' facilities in surrounding towns to cope with the expected interest from astro-tourists; science centres; development of curriculum material related to the SKA; the inspiration of popular culture, etc. Further afield, local opportunities will arise around the users' home institutions in 
response to the work they will be undertaking remotely with the telescopes, ensuring that SKA has a truly global presence. The potential reach of the SKA in terms of skills development is therefore enormous.

The SKA will need thousands of people from across many countries in a range of professions to design, build and operate it and to run all the associated services. Builders, caterers and administrative staff as well as scientists, engineers, IT specialists, communicators, etc. are already being employed in this endeavour, encouraging the creation or enhancement of a broad range of training and skills development opportunities. Of particular interest both for the immediate and specific needs of the SKA and for the future extension of knowledge-based economies is the development of STEM skillsets.

Sustainability is also about skilled people, both supporting the project's implementation, and arising from the project's implementation. To ensure a sufficiently large pool of trained scientists, engineers and technicians by the time the SKA is ready to be operated, it is urgent to inspire young people now. By working closely with education authorities as early as possible, building on the experience and success of existing efforts, in particular NASA/HST or astronomy education programmes such as Universe Awareness (UNAWE), the SKA can make a substantial contribution to current efforts to bring astronomy onto the school curriculum in a number of countries. The SKA Organisation and its Members are already actively engaged in developing programmes in pursuit of these aims. The mounting concerns about the widespread shortage of trained scientists, engineers and technicians are not limited to the SKA and its own needs. These skills are critical to countries' increasingly technologically dependent economies, and governments are keen to find ways to promote them. The active engagement of the SKA Organisation/SKA Observatory IGO in generating wider interest in and support for training in these subjects, including strengthening recognition of the need for and value of them even among those who will not directly follow this path, will contribute to wider economic advancement as well as meeting its own specific needs. The SKA can become the first international scientific infrastructure with education and outreach embedded in its development from the earliest stages, inspiring young people in time for them to become users of the telescope or engineers and scientists working with the SKA, and potentially encouraging more people to develop he STEM skills needed to maintain and grow the knowledge-based economy.

\section{Case Study: SKA South Africa HCD Programme}

In 2005, South Africa established a Human Capital Development (HCD) Programme to create capacity in relevant radio astronomy science and engineering. The programme, which is available to South Africans, and to students from SKA SA Partner Countries in Africa, provides support at all academic levels, to ensure a continuous throughput of young people moving into relevant studies and research, and to ensure the required supervisory and teaching capacity is in place to support 
the students. To date, the programme has awarded more than several hundred grants to students and universities. In addition to the research capacity development aspects of the programme, SKA SA has also focused on developing skills for the operations and maintenance of the MeerKAT facility. The Artisan and Technician Training programmes have well defined schedules of the numbers of technically skilled staff required, and are on target to meet the capacity needs. In the towns that are close to the SKA SA site in the Karoo, the SKA SA HCD programme has facilitated the recruitment and secondment of Mathematics and Science teachers in the schools, provided bursaries for learners to attend Carnarvon High School (in the town nearest the site), rolled out cyberlabs and E-learning centres (in cooperation with the Departments of Education and Rural Development, and with industry partners), and trained local residents to manage cyber-centres, which are available to members of the community.

\section{Conclusion}

Sustainability for a research infrastructure means being able to motivate a continued significant investment to support activities. It requires a multidisciplinary approach which brings together a suitable governing legal framework, a policy environment which permits financial stability, all underpinned by a scientific and socio-economic case. SKA is working hard in all domains, with many aspects to come together with the formation of the SKA Observatory IGO and approval of the telescope's construction in 2020 .

\section{Full Presentation}

https://indico.cern.ch/event/727555/contributions/3461263/attachments/1868120/ 3072746/FCCW_0900_Berry_SKA_approach_to_sustainable_research.pdf

Open Access This chapter is licensed under the terms of the Creative Commons Attribution 4.0 International License (http://creativecommons.org/licenses/by/4.0/), which permits use, sharing, adaptation, distribution and reproduction in any medium or format, as long as you give appropriate credit to the original author(s) and the source, provide a link to the Creative Commons licence and indicate if changes were made.

The images or other third party material in this chapter are included in the chapter's Creative Commons licence, unless indicated otherwise in a credit line to the material. If material is not included in the chapter's Creative Commons licence and your intended use is not permitted by statutory regulation or exceeds the permitted use, you will need to obtain permission directly from the copyright holder.

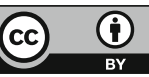

